\title{
A comparative study on the dynamics of high speed spindles with respect to different preload mechanisms
}

\author{
Hongrui Cao • Tomas Holkup • Yusuf Altintas
}

Received: 18 May 2010/Accepted: 24 April 2011 /Published online: 4 May 2011

(C) Springer-Verlag London Limited 2011

\begin{abstract}
This paper presents the effects of bearing preload mechanisms on the dynamic performance of high speed spindles. The comparisons of two main types of bearing preload - "constant" and "rigid"-mechanisms are provided using a mathematical model as well as experiments. Based on the Timoshenko beam element theory coupled with a nonlinear model of angular contact ball bearings, the dynamics of the spindle shaft, housing, and bearings system is modeled as a nonlinear function of preload mechanism and amplitude, spindle speed, and external cutting loads. The mathematical model of the spindle is experimentally validated by comparing the predicted and measured static displacements, mode shapes, frequency response functions, and natural frequencies under different conditions. The performance of spindles under rigid and constant force preload is investigated systematically using a mathematical model under various conditions. It is shown, among other things, that at high speeds and under cutting loads the rigid preload mechanism is more efficient in maintaining the dynamic stiffness of spindles than constant preload.
\end{abstract}

Keywords Spindle $\cdot$ Preload $\cdot$ Bearing $\cdot$ Dynamics

H. Cao

State Key Laboratory for Manufacturing Systems Engineering,

Xi'an Jiaotong University,

Xi'an 710049, People's Republic of China

H. Cao $(\bowtie) \cdot$ T. Holkup • Y. Altintas

Manufacturing Automation Laboratory, Department of

Mechanical Engineering, The University of British Columbia,

Vancouver, BC V6T 1Z4, Canada

e-mail: chr@mail.xjtu.edu.cn

URL: www.mech.ubc.ca/ mal

$\begin{aligned} & \text { Nomenclature } \\ & \{x\},\{\dot{x}\},\{\dot{x}\}\end{aligned}$
$\{q\}$
$\delta, \gamma$
$[M],[K],[C],[G]$
$\Omega$
$\{F\},\{R\}$
$\{\Delta x\}$
$\Delta E$

Displacement velocity and acceleration vectors

Motion vector of the node

Translational and rotational displacements

Mass stiffness, damping and gyroscopic matrices

Spindle rotating speed

External and unbalance forces, respectively

Incremental displacement vector

Unbalance energy of the system

\section{Superscript \\ i,o Inner ring and outer ring \\ s,h Shaft and housing}

\section{Subscript \\ S,B Shaft and bearing \\ C Centrifugal effects \\ D Structural damping \\ $x, y, z \quad$ Cartesian coordinates}

\section{Introduction}

High speed spindles are widely used in the aerospace industry for machining aluminum alloys, and in the die and mold industry for finishing hardened steel alloys. Apart from excessive tool wear, which can be minimized by selecting suitable cutting speeds and tool materials, chatter vibrations are the biggest obstacle in increasing material removal rates in high speed machining. The spindle is one of the key components that limit the overall dynamic stiffness of the machine tool. If the 
system's frequency response function (FRF) is known at the tool tip, the chatter free cutting conditions can be predicted to achieve optimal metal removal rates without damaging the machine tool and the workpiece [1]. In order to design, analyze, and test spindles in a virtual environment before resorting to costly physical trials, accurate modeling of spindle dynamics is essential [2].

The rotating shaft is attached to the housing with angular contact ball bearings, which constitute the most critical components of the spindle system. Proper preload of angular contact bearings is very important for the rigidity, rotating accuracy, and bearing life of spindles. There are three main types of bearing preload mechanisms used in practice: rigid preload, stiff spring preload, and constant preload [3]. Rigid preload, also called lockring preload or fixed position preload, is applied by inducing a constant relative displacement between the inner ring and the outer ring by using unevenly ground spacers or match-ground bearings. However, thermal deformation of spindle parts can change the relative position of bearing rings and severely affect the preload of bearings. In order to mitigate the thermal effects on bearing preload, springs can be used to absorb the thermal expansion. If the spring stiffness cannot be ignored (stiff spring preload case), the extra preload force is equal to the product of spring stiffness and outer ring thermal displacement. Furthermore, to maintain the preload constant, corresponding designs - so-called constant preload or constant-force preload mechanism using hydraulic systems or soft springs - have been developed $[4,5]$. The dynamic behavior of the spindle system is quite different under various bearing preload mechanisms. The rigid preload mechanism is simple and practical to implement. However, the preload force can change significantly due to relative thermal deformation between the spindle shaft and housing, which may result in excessive preload and seizure of the bearings during high-speed cutting. Although overpreload can be avoided effectively in stiff spring preload or constant preload mechanisms, the spindle rigidity may not be maintained at desired levels for heavy cutting operations $[5,6]$. As a result, designers mostly rely on their experience when selecting the preload mechanism and magnitude for safe and optimal operations of spindles in industry.

Considerable research related to bearing preload issues have been carried out, which includes thermally induced bearing preload [7-9], bearing preload monitoring [10, 11], control [6, 12], and optimization [13]. However, few investigations have been performed on sensitivity of spindle dynamics to different bearing preload mechanisms. $\mathrm{Li}$ and Shin presented the effects of bearing configuration on the dynamic stiffness and thermal behavior of high speed spindles, using numerical simulations [3]. Similarly, Lin et al. used their model to predict the properties of a spindle with bearings preloaded by two different mechanisms [14]. Cao and Altintas [15] presented a dynamic model of spindles with constant preload; the effects of preload magnitude on bearing stiffness and spindle dynamics have been investigated in detail. However, they did not consider the rigid preload case.

This paper presents a coupled model of spindle-bearing systems improved by adding the rigid preload mechanism to the previous model developed by Cao and Altintas [15]. Various results such as bearing contact forces, angles and stiffness matrices, spindle static deformations, natural frequencies, and FRFs can be obtained as output of the model under specified preload, spindle speed, and cutting loads. The model is verified on an experimental spindle under static as well as rotating conditions. Subsequently, the dynamic behavior of spindle with rigid and constant preload mechanisms is compared systematically under various operating conditions.

\section{Modeling a spindle-bearing system}

The spindle shaft/housing and the angular contact ball bearing are modeled separately, and new possibilities of their integration allow considering different preload mechanisms other than constant preload.

\subsection{Spindle shaft/housing model}

The spindle shaft/housing is modeled using the Timoshenko beam element-based finite element (FE) method. The motion of each FE node is described by three translational $\left(\delta_{x}, \delta_{y}, \delta_{z}\right)$ and two rotational $\left(\gamma_{y}, \gamma_{z}\right)$ degrees of freedom:

$$
\{q\}=\left\{\delta_{x}, \delta_{y}, \delta_{z}, \gamma_{y}, \gamma_{z}\right\}^{T}
$$

where torsional motion is not included. The equation of motion for the spindle shaft and housing is expressed by including the centrifugal force and gyroscopic effects as

$$
[M]_{\mathrm{S}}\{\ddot{x}\}-\Omega[G]_{\mathrm{S}}\{\dot{x}\}+\left([K]_{\mathrm{S}}-\Omega^{2}[M]_{\mathrm{C}}\right)\{x\}=\{F\}
$$

where $[K]_{\mathrm{S}},[M]_{\mathrm{S}}$ and $[G]_{\mathrm{S}}$ are the stiffness, mass and skewsymmetric gyroscopic matrices of the spindle shaft and housing. $[M]_{\mathrm{C}}$ is the mass matrix for the centrifugal force effect, and $\{F\}$ is the external force vector. The damping matrix is not included in the FE model of the shaft/housing. The rotating speed $\Omega$ is set to zero when modeling the spindle housing. Besides, pulleys or other disk-like accessories are modeled as rigid mass elements.

\subsection{A coupled bearing model with different preload mechanisms}

The coupling between the spindle shaft/housing and bearing structures is modeled as shown in Fig. 1, where the bearing, shaft, and housing are integrated into a single 


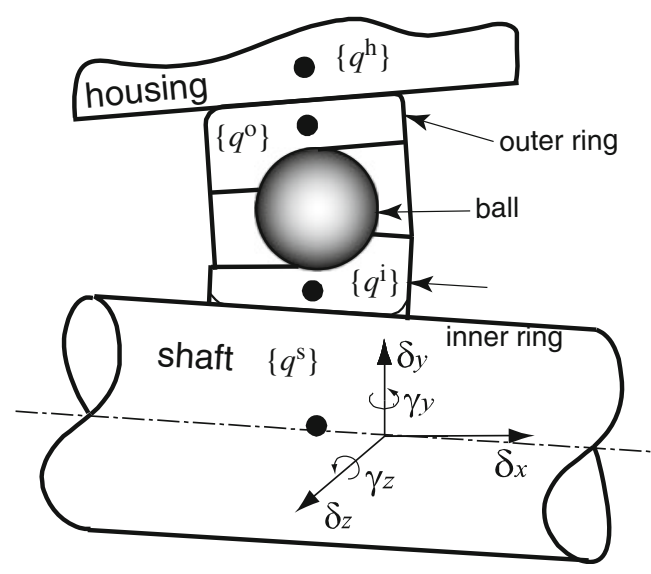

Fig. 1 Coupled shaft-bearing-housing model

elastic system. The black dots represent nodes. Each bearing element consists of two nodes - the inner ring node and the outer ring node-which are coincident with the nodes on the shaft and housing, respectively. As a result, the rings have the same $\left\{q^{\mathrm{i}}\right\}$ (inner ring and shaft) and $\left\{q^{\circ}\right\}$ (outer ring and housing) motions. The nodes representing the spindle housing can be either left free for modeling the spindle in a free-free state or connected to structures representing the dynamics of the machine tool; details have been published by Cao and Altintas [16]. Furthermore, the dynamic model of the spindle can be extended to include thermal deformations once these are computed separately by a specialized model of heat transfer and thermal deformations.

The displacement relations among the spindle shaft, bearings, and the housing correspond to the configuration and preload mechanism of bearings. Given the same bearing configuration, the only difference among these three preload mechanisms, i.e., rigid preload, constant preload, and stiff spring preload, is the boundary condition in the axial direction. Since the stiff spring preload system is the least common of the three, only rigid preload and constant preload are studied in detail.

An O-type bearing configuration with rigid and constant preload mechanisms is shown in Fig. 2 as an example. The two bearings belong to one force chain and the system is self-balanced in the axial direction under the preload. Regardless of the preload type used, all the inner rings are fixed to the shaft in this two-bearing system:

$$
\left\{q^{\mathrm{i}}\right\}=\left\{q^{\mathrm{s}}\right\}
$$

The connections between the outer rings and the housing are different in the two preload cases. In the rigid preload case, the outer rings of both front and rear bearings are fixed to the housing with a constraint equation including a constant term $\delta_{a 0}$ in the axial direction. The constant term corresponds to the designed preloading difference in outer/ inner assembly lengths. The displacement relation of the outer ring and a corresponding housing node is coupled using the equation:

$$
\left\{q^{\mathrm{o}}\right\}=\left\{q^{\mathrm{h}}\right\}+\left\{\delta_{a 0}, 0,0,0,0\right\}^{T}
$$

Correspondingly, constant preload provided by a soft spring acts on the outer ring of the rear bearing in the constant preload case. The constant preload force is used as an input boundary condition. Contrary to rigid preload, the outer ring of the rear bearing is free in the axial direction and the displacement relationship between the rear bearing and the housing is:

$\delta_{y}^{o}=\delta_{y}^{h} \delta_{z}^{o}=\delta_{z}^{h} \gamma_{y}^{o}=\gamma_{y}^{h} \gamma_{z}^{o}=\gamma_{z}^{h}$

The coupled bearing model allows the inclusion of additional deformations related to the spindle shaft and housing (e.g., radial press-fit mounting, thermal expansion if known) by using Eqs. 3, 4, and 5. The displacements of the shaft and housing due to external forces can be obtained by solving the global equation of motion which is presented in the following section.

\subsection{Global equations of motion of the spindle-bearing system}

By assembling the equations of spindle shaft/housing and bearings, the following general nonlinear equilibrium equation for the spindle-bearing system is obtained:

$$
[M]\{\ddot{x}\}+[C]\{\dot{x}\}+[K(x)]\{x\}=\{F\}
$$

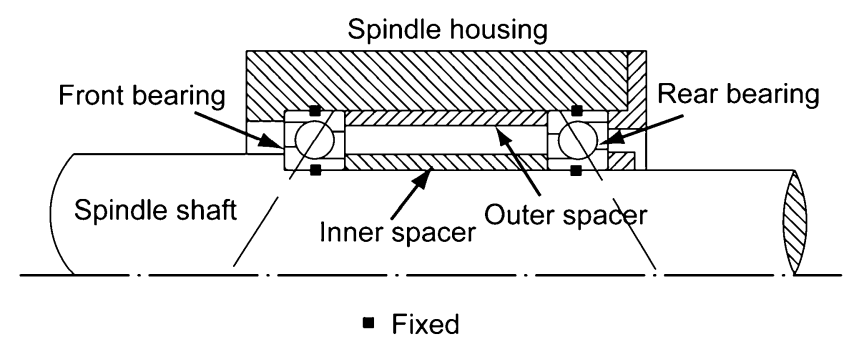

(a) Rigid preload

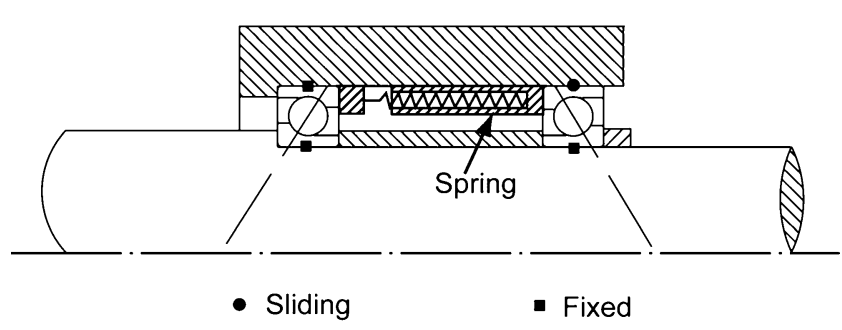

(b) Constant preload

Fig. 2 Bearing preload mechanisms 
where, $[M]=[M]_{\mathrm{S}},[C]=[C]_{\mathrm{D}}-\Omega[G]_{\mathrm{S}}, \quad[K(x)]=[K]_{\mathrm{S}}+$ $[K(x)]_{\mathrm{B}}-\Omega^{2}[M]_{\mathrm{C}}$ are the mass, damping, and stiffness matrices. $[C]_{\mathrm{D}}$ is the structural damping matrix constructed from modal damping ratios identified experimentally from similar spindles. The bearing stiffness matrix $[K(x)]_{\mathrm{B}}$ depends on displacement $\{x\}$, among other factors. In turn, the displacement is affected by the system stiffness and the external force. Therefore, the dependency of bearing stiffness matrices on displacement is the root cause of the nonlinearity in the spindle system model. The external force $\{F\}$ is determined by the cutting conditions. The details of the FE matrices and formulations have been previously presented in [15], and will not be repeated here. The noted contribution is the identification of the bearing stiffness matrix $[K(x)]_{\mathrm{B}}$ which is found iteratively using the Newton-Raphson method as follows.

The static external force case is considered first and the iteration equations are given as

$$
\begin{aligned}
& {[M]\{\ddot{x}\}^{i}+[C]\{\dot{x}\}^{i}+[K]^{i}\{x\}^{i}=\{F\}} \\
& {[K]^{i}=[K]_{\mathrm{S}}+[K]_{\mathrm{B}}^{i}-\Omega^{2}[M]_{\mathrm{C}}} \\
& \{x\}^{i}=\{x\}^{i-1}+\{\Delta x\}^{i}
\end{aligned}
$$

where, $i$ is the iteration index.

The iteration starts with an initially assumed bearing stiffness $[K]_{\mathrm{B}}^{0}$; the initial stiffness of the spindle system is then obtained as:

$$
[K]^{0}=[K]_{\mathrm{S}}+[K]_{\mathrm{B}}^{0}-\Omega^{2}[M]_{\mathrm{C}}
$$

Under static external loads, the velocity $\{\dot{x}\}^{i}$ and acceleration $\{\ddot{x}\}^{i}$ are both 0 . The initial displacement vector $\{x\}^{0}$ of the iteration depends on the preload type. For rigid preload, the initial displacement of the spindle system is the sum of the displacement caused by the static external force $\{F\}_{0}$ and a constant vector $\{x\}_{\text {pre }}$ which includes information about the position preload of bearings:

$\{x\}^{0}=\left([K]^{0}\right)^{-1}\{F\}_{0}+\{x\}_{\text {pre }}$

In the constant preload case, the initial displacement $\{x\}^{0}$ is obtained using the initial system stiffness $[K]^{0}$ and the resultant load of the external force $\{F\}_{0}$ and the preload force $\{F\}_{\text {pre }}$ :

$\{x\}^{0}=\left([K]^{0}\right)^{-1}\left(\{F\}_{0}+\{F\}_{\text {pre }}\right)$

In the $i^{\text {th }}(i \geq 1, i \in Z)$ step of the iteration, the bearing stiffness $[K]_{\mathrm{B}}^{i}$ is evaluated through Jones' bearing model [17] by using the displacement $\{x\}^{i-1}$ of the previous step, and the system stiffness matrix $[K]^{i}$ is then updated by using Eq. 8. The unbalance force $\{R\}^{i}$ of the $i^{\text {th }}$ iteration step is evaluated as:

$\{R\}^{i}=\{F\}-[K]^{i}\{x\}^{i-1}$

where, $\{F\}=\{F\}_{0}$ in the rigid preload case, while $\{F\}=\{F\}_{0}+\{F\}_{\text {pre }}$ in the constant preload case. The unbalance force creates the incremental displacement:

$\{\Delta x\}^{i}=\left([K]^{i}\right)^{-1}\{R\}^{i}$

Adding the incremental displacement $\{\Delta x\}^{i}$ to the displacement $\{x\}^{i-1}$, the displacement vector $\{x\}^{i}$ is updated by using Eq. 9. The updated displacement vector $\{x\}^{i}$ is used to obtain the new bearing stiffness matrices of the next iteration. The unbalance energy of the system at the $i^{\text {th }}$ step is defined as,

$\Delta E^{i}=\left(\{R\}^{i}\right)^{T}\{\Delta x\}^{i}$

which is used as a convergence criterion for the iteration. The iteration process is repeated until the unbalance energy $\Delta E^{i}$ is smaller than or equal to a set limit value.

The effects of time-varying external loads $\{F(t)\}$, e.g., periodic milling forces, on the bearing stiffness can also be considered. Then the system responses and the bearing stiffness are time varying and the equation of motion at time $t+\Delta t$ is.

$$
[M]\{\ddot{x}\}_{t+\Delta t}+[C]\{\dot{x}\}_{t+\Delta t}+[K(x)]_{t+\Delta t}\{x\}_{t+\Delta t}=\{F\}_{t+\Delta t}
$$

Assuming that the displacement, velocity, and acceleration vectors at time $t(t \geq 0)$ are known, the time history responses of the spindle system (i.e., displacement $\{x\}_{t+\Delta t}$, velocity $\{\dot{x}\}_{t+\Delta t}$ and acceleration $\{x\}_{t+\Delta t}$ ) at time $t+\Delta t$ can be evaluated numerically using the Newmark integration method. Then the bearing stiffness of each time step can be found using the same iteration method (Eqs. 7, 8, and 9) as described above.

\section{Experimental verification}

The dynamic model of the spindle-bearing system has been validated through static and dynamic response tests on an experimental spindle shown in Fig. 3. The spindle has two front bearings GMN-HYKH61914 and three rear bearings GMN-HYKH61911 in an O-type configuration. Constant and rigid bearing preloads are applied as follows. The constant preload mechanism is implemented by applying hydraulic oil pressure on the floating hydraulic sleeve of the rear bearing. The hydraulic force is then transferred to the rear bearings, shaft, and front bearings. After the whole 
Fig. 3 Experimental spindle instrumented with displacement sensors and preload application mechanism

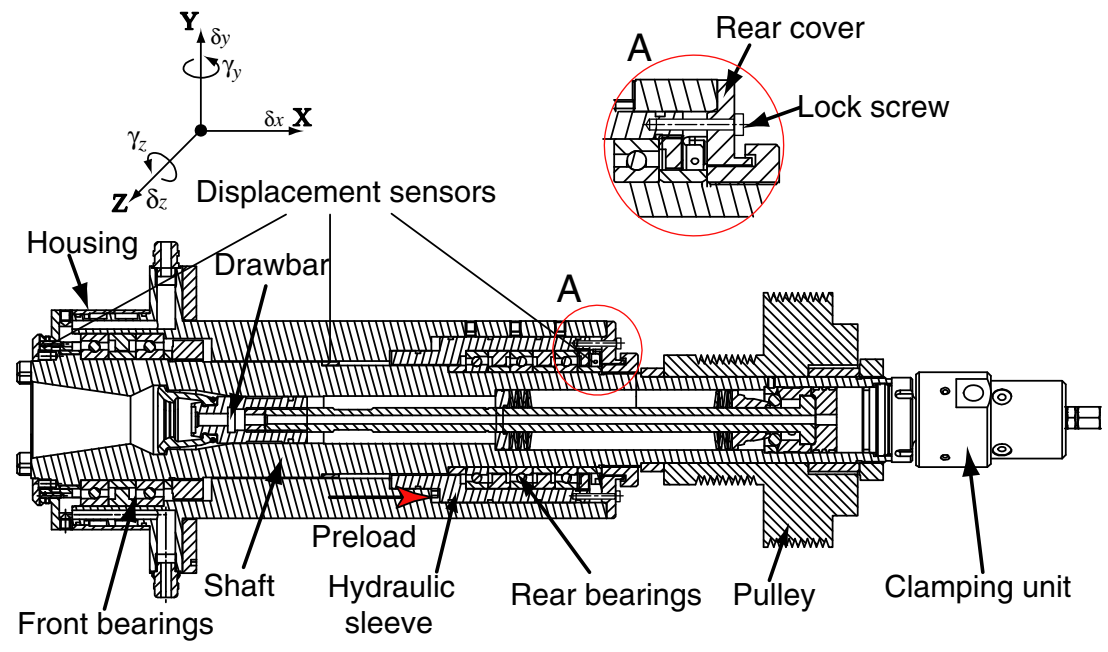

system reaches a self-balanced state in the axial direction, both front and rear bearings are preloaded. This constant preload system can be switched to the rigid preload mechanism easily on the test spindle: the hydraulic sleeve is first pushed to the desired position by hydraulic oil, and then fastened gently to the rear spindle cover using lock screws. The hydraulic force is released in the end; the force chain is the same as in the constant preload case.

Static test Axial preload forces were applied to the hydraulic sleeve incrementally while the spindle housing was fixed to a bench. The axial displacements of the spindle nose and hydraulic sleeve were measured by a laser displacement sensor and a contact LVDT displacement sensor, respectively, starting with an initial preload of $280 \mathrm{~N}$. The experimental displacement measurements confirm the proposed FE model of the spindle as shown in Fig. 4. The displacement of the hydraulic sleeve is approximately twice as large as the displacement of the spindle nose.

Dynamic response test The pulley and the clamping units were disassembled during the modal tests to focus on the

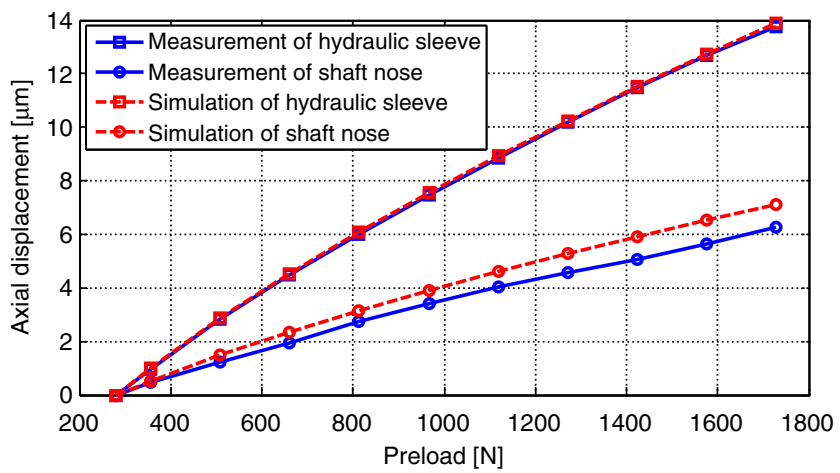

Fig. 4 Simulated and experimentally measured displacements in the axial direction (a) Constant preload
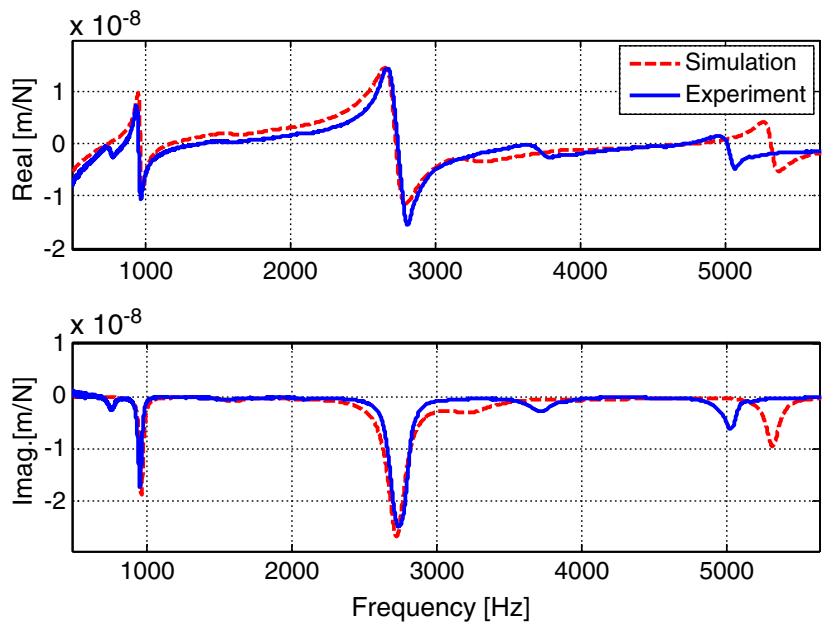

(b) Rigid preload
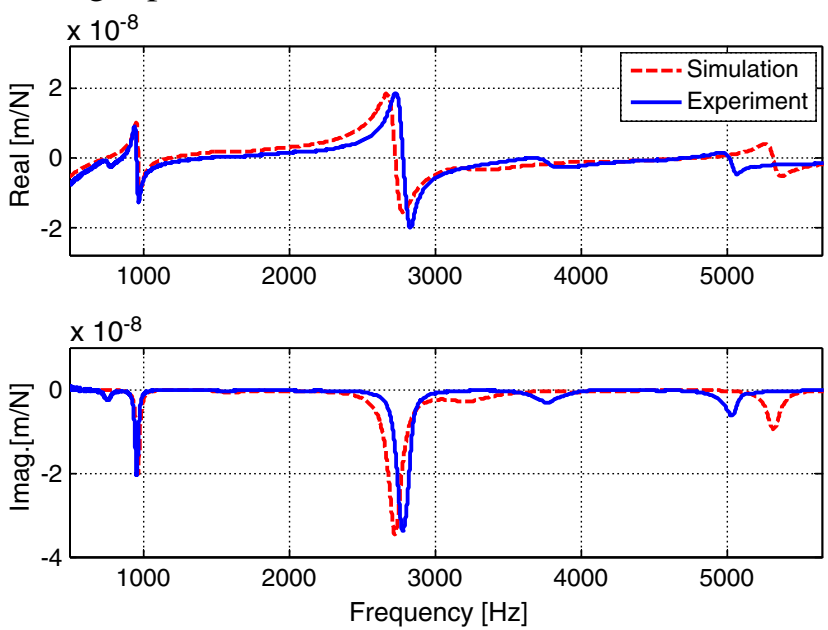

Fig. 5 Simulated and experimentally measured FRFs at the spindle nose in the free-free conditon (bearing preload: $815 \mathrm{~N}$ ) 


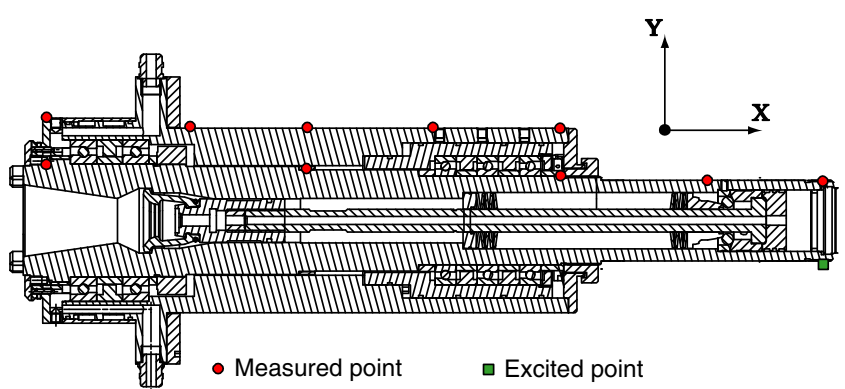

Fig. 6 Layout of the modal tests

shaft-bearing-housing assembly. The FRFs of the spindle in the radial directions were measured by applying an impact force on the spindle nose and recording the vibration response at the opposite part of the nose. The measurements were made at the spindle nose under free-free conditions by CutProMalTF ${ }^{\circledR}$ Fourier Analyzer. The hydraulic preload was set to $815 \mathrm{~N}$ for the constant preload mechanism, and an equivalent preload value was ensured in the rigid preload case. The simulated and experimentally measured FRFs at the spindle nose are shown in Fig. 5. The modal damping ratios in different preload cases were borrowed from experimental data and used in the FE simulations. From the simulated FRFs, the first mode $\left(\omega_{n 1}^{s}=965 \mathrm{~Hz}\right)$ and fourth mode $\left(\omega_{n 4}^{s}=\right.$ $2,722 \mathrm{~Hz}$ ) have been found to be the most dominant, having the greatest effect on the stability of machining operations. Corresponding to these two main modes, experimental model damping ratios of $1.6 \%\left(\omega_{n 1}^{x}=955 \mathrm{~Hz}\right)$ and $2.9 \%\left(\omega_{n 4}^{x}=\right.$ $2,745 \mathrm{~Hz})$ were used in the simulation of the constant preload case, while $1.4 \%\left(\omega_{n 1}^{x}=955 \mathrm{~Hz}\right)$ and $2.0 \%$ $\left(\omega_{n 4}^{x}=2,778 \mathrm{~Hz}\right)$ damping ratios were used in the rigid preload case. Damping ratios of other modes were set to $3 \%$.

Theoretically, the radial FRFs are almost identical, even though the mathematical models are slightly different for the rigid and constant preload of the spindle bearings when the equivalent preload forces are the same and the nonlinear effects caused by the impact force are neglected. However, with the sleeve fastened to the spindle cover plate with lock screws, it is difficult to obtain a totally equivalent rigid

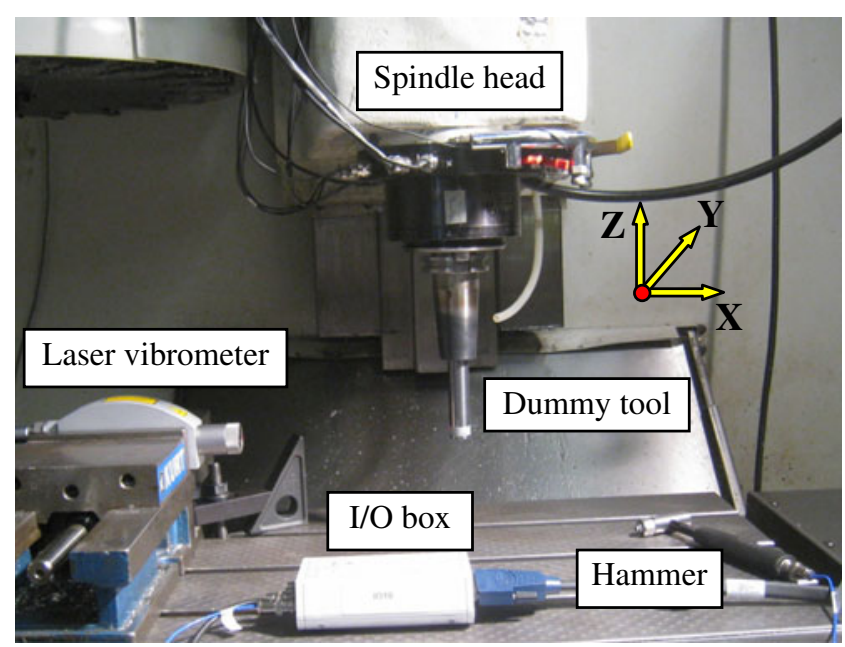

Fig. 8 Experimental setup

connection; therefore, the fourth mode of the rigid preload case shifts slightly to a higher frequency with decreased system damping ratios during the experiments. Generally, the simulated and experimental FRFs are in reasonable agreement, which indicates the validity of the proposed bearing preload models.

Modal analysis Mode shape tests of the spindle under freefree conditions have been carried out to identify the vibration modes. The excitation point was placed at the rear part of the spindle shaft, while multiple measurement points were distributed on the shaft and housing, as shown in Fig. 6.

The mode shapes are shown to be identical for both constant and rigid preload mechanisms. The simulated and experimentally measured mode shapes of the two dominant modes are shown in Fig. 7. The mode at $965 \mathrm{~Hz}$ is dominated by the first flexural mode of the shaft with little influence from the bearings, while the fourth bending mode at $2,722 \mathrm{~Hz}$ reflects the influence of bearings and the flexural mode of the shaft. This observation has also been demonstrated by Cao and Altintas [15], where the increased preload has been shown not to affect the first mode, while
Fig. 7 Simulated and experimental mode shapes
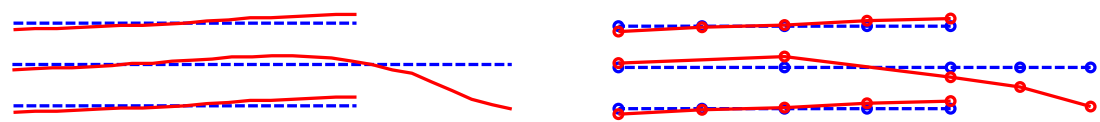

(a) $1^{\text {st }}$ mode $(965 \mathrm{~Hz})$

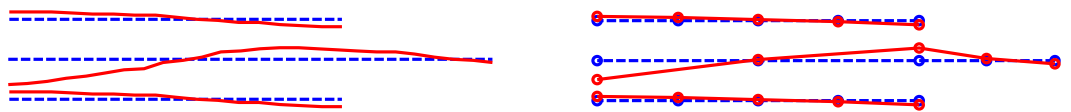

(b) $4^{\text {th }}$ mode $(2722 \mathrm{~Hz})$ 


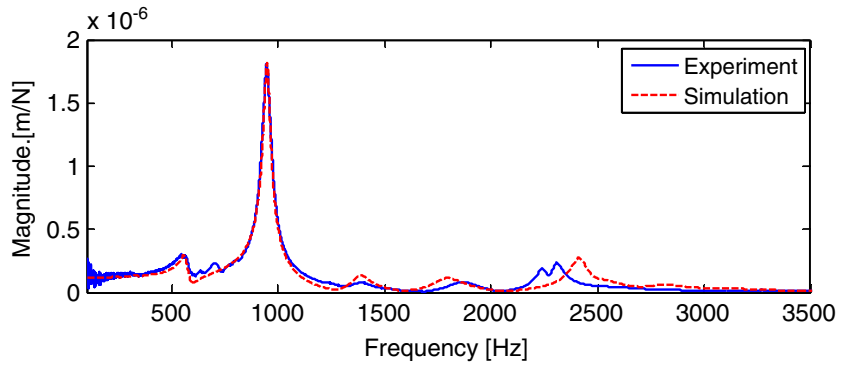

(a) X direction

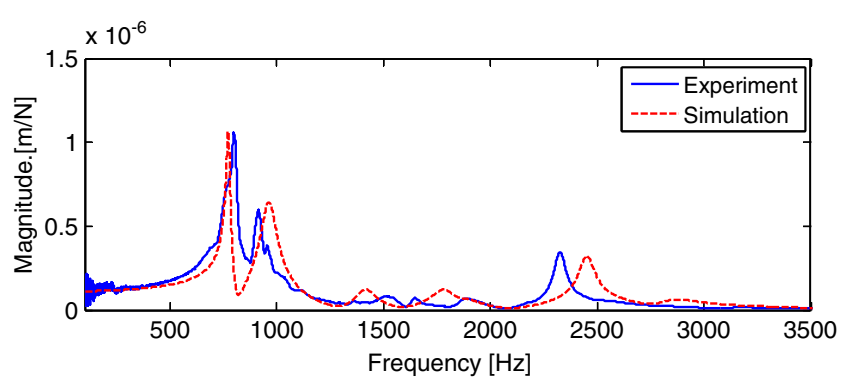

(b) Y direction

Fig. 9 Simulated and experimental FRFs at the dummy tool tip in the stationary condition (constant preload: 1,728 N)

shifting the fourth mode upward. The simulations match well with the experimental results, which confirm the accuracy of the proposed dynamic model of the spindle.

Dynamic test after mounting on the machine tool Due to the effects of the machine tool, the dynamics of the spindle changes after mounting. Detailed modeling of the spindle and machine tool system is described in [16]. There, the FE model of the full spindle assembly including the pulley, the clamping unit, and the spindle head has been verified in both stationary and running states. The experimental setup is shown in Fig. 8. The FRFs at the tip of the dummy tool were measured using a laser vibrometer and a modal hammer.

Due to the unsymmetric shape of the spindle head, the dynamics of the spindle system in both the $X$ and $Y$ directions need to be validated. Figure 9 shows the FRFs at the tool tip in the stationary state where a constant hydraulic preload of $1,728 \mathrm{~N}$ is applied. It can be seen that the simulations match with the experimental results very well in both the $X$ (Fig. 9a) and $Y$ (Fig. 9b) directions and the rigidity of the spindle system is higher in the $\mathrm{Y}$ direction.

Tests were also conducted to measure the FRFs at the dummy tool tip under various spindle speeds. The rotating speed was increased from 0 to $12,000 \mathrm{rev} / \mathrm{min}$ with an increment of $1,000 \mathrm{rev} / \mathrm{min}$ and the same constant hydraulic preload of $1,728 \mathrm{~N}$ was kept in each speed step. Since the hydraulic preloaded bearings can accommodate thermally induced preload and the measurement time is very short at each rotational speed step, the thermal effects have been assumed to be negligible during the experiment. In order to simplify the problem, only the spindle dynamics in the $X$ direction are presented here. From Fig. 9a, it can be seen that the three dominant frequencies below $3,500 \mathrm{~Hz}$ are around 565, 960, and 2,320 Hz. However, under high-speed rotating conditions, the peaks at $2,320 \mathrm{~Hz}$ are too small to observe. The natural frequencies of the first two dominant modes $(565,960 \mathrm{~Hz})$ over the speed range of $0-12,000 \mathrm{rev} /$ min are shown in Fig. 10. Due to the influence of the centrifugal force and the gyroscopic moment acting on the spindle shaft and bearings, the natural frequencies of both modes decrease with spindle speed [15]. "Simulation 1" and "simulation 2" are the forward and backward natural frequencies caused by the gyroscopic effect of the spindle shaft, respectively. However, the experimental natural frequencies do not have these two branches. One possible reason is that the existing out-of-balance excitation can only excite resonance in a forward whirl mode due to the

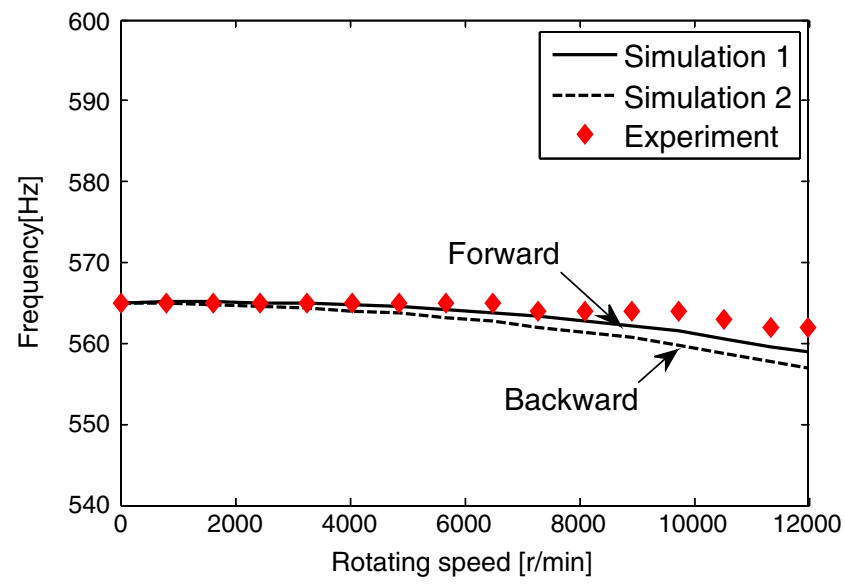

(a) The first dominant mode

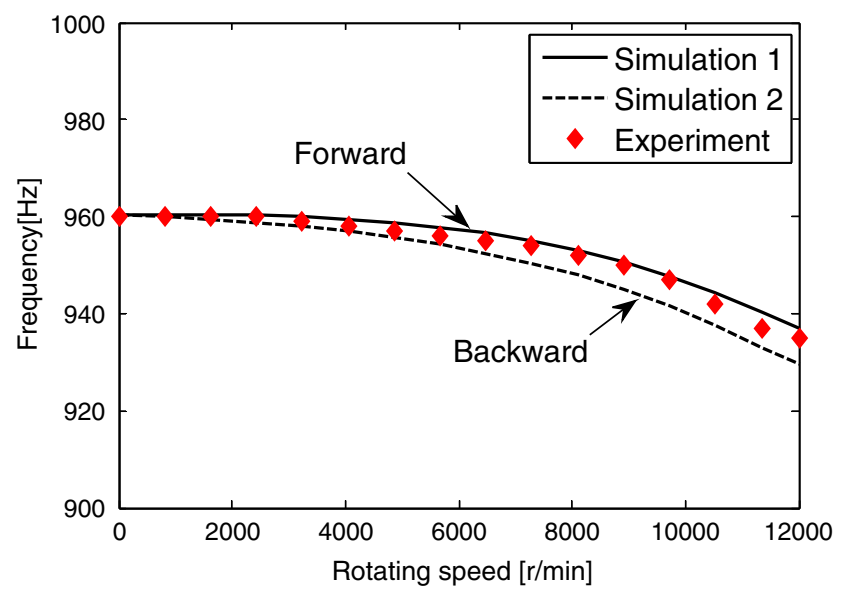

(b) The second dominant mode

Fig. 10 The natural frequencies of the spindle under various speeds (constant preload: 1,728 N) 


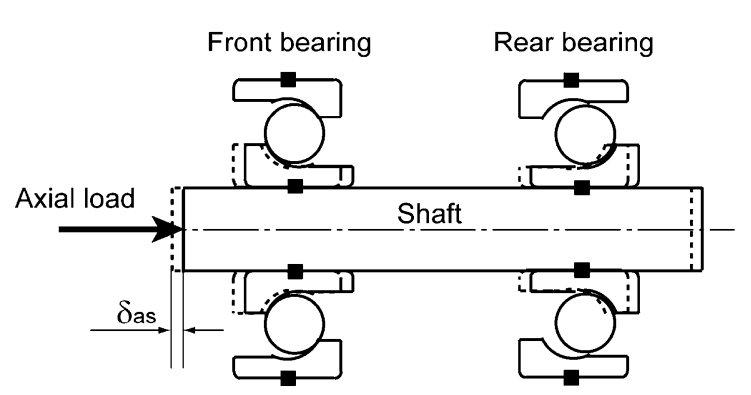

(a) Rigid preload

With axial load

$\delta$ as: The axial displacement of shaft

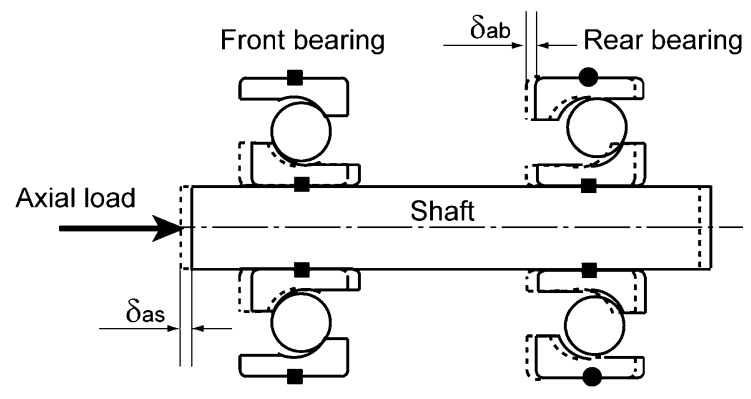

(b) Constant preload

\section{....... Without axial load}

$\delta a b:$ The axial displacement of rear bearing

Fig. 11 A two-bearing example - motions of shaft and bearings due to axial load

symmetrical properties of all the bearings [18]; another reason may be that the forward and backward modes are closely coupled and the average of the two frequencies is obtained from multiple measurements [19]. Overall, the comparison in Fig. 10 shows that predicted natural frequencies agree very well with the experimental results over the entire speed range.

\section{Analytical comparisons of preload mechanisms}

The static and dynamic properties of the experimental spindle with both constant and rigid preload mechanisms have been predicted using the presented analytical model and compared under various operating conditions. The results are summarized as follows.

\subsection{Axial load effects}

The external axial loads are applied at the spindle nose in the $X$ direction (seen in Fig. 3) and the initial preload forces for

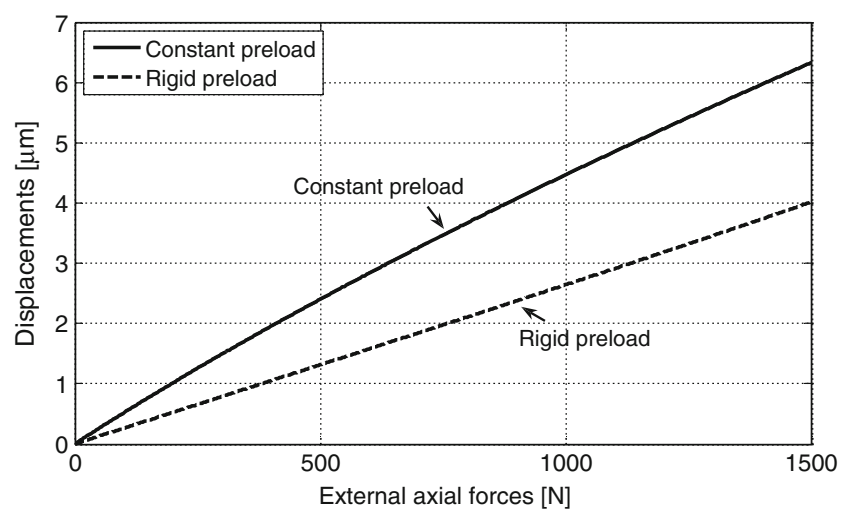

Fig. 12 Axial displacements at the spindle nose under axial loads (bearing preload: $720 \mathrm{~N}$, stationary state) both preload cases are set to $720 \mathrm{~N}$. For the experimental spindle with an O-configuration, the load on the front bearings increases with external forces in both preload cases. The preload of the rear bearings decreases in the rigid preload case, while they maintain the stiffness in the constant preload case, since the outer ring can slide with the shaft. The motions of the shaft and the bearing rings due to the axial load are demonstrated using a two-bearing system as shown in Fig. 11.

The displacements of the spindle nose under external axial loads are shown in Fig. 12. The displacements in both preload cases increase with the axial loads, and the displacement of the constant preload system is much larger.

The variation of axial rigidity with increasing external axial load in the stationary state is shown in Fig. 13. As only the front bearings contribute to the axial stiffness of the whole spindle system in the constant preload case, the overall axial rigidity of the rigid preload system is about twice as large as that of the constant preload system when the external axial load is absent. As the load increases, the axial rigidity of the constant preload system increases, while the rigid preload case shows an opposite trend.

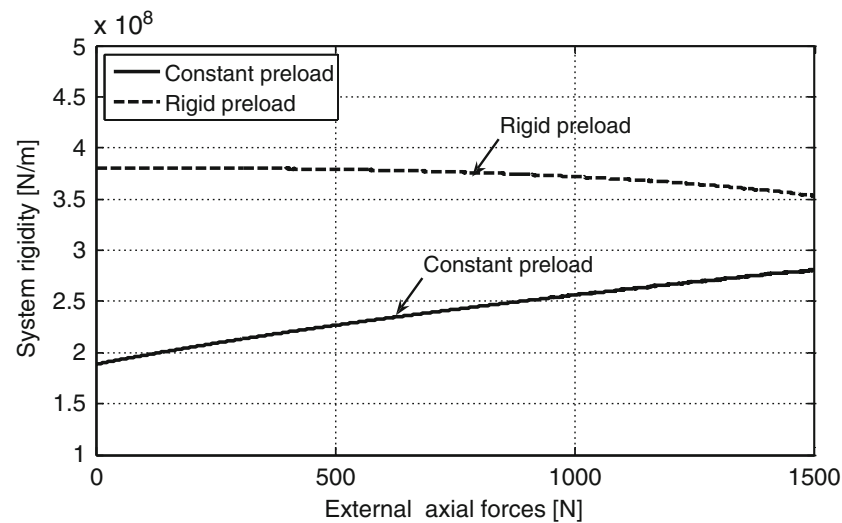

Fig. 13 System rigidity in the axial direction under axial loads (bearing preload: $720 \mathrm{~N}$, stationary state) 

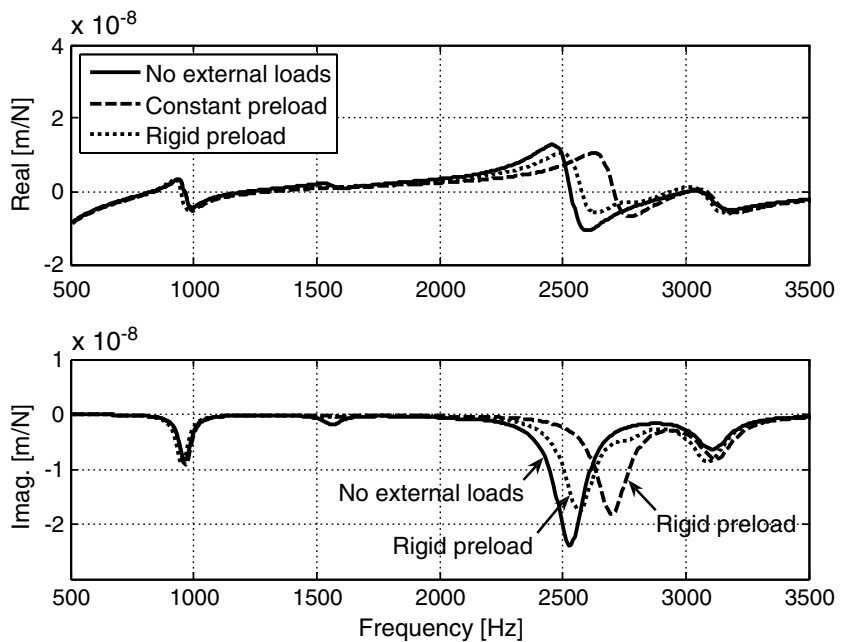

Fig. 14 FRFs at the spindle nose in the radial direction (bearing preload: $720 \mathrm{~N}$; external axial load: $750 \mathrm{~N}$, stationary state)

The FRFs at the spindle nose in the radial direction are predicted in Fig. 14 with an external axial load of $750 \mathrm{~N}$. Without external loads, FRFs of both preload cases are almost identical. When the axial load increases, natural frequencies of the spindle in both preload cases increase due to the increased stiffness of the front bearings; however, the external axial load has smaller effect on the rigid preload system.

\subsection{Radial load effects}

As spindle systems are axisymmetric, the external radial load has only been applied in one direction at the spindle nose during simulations. In both cases, the initial preload forces have been set to $720 \mathrm{~N}$. Besides radial displacement, the radial force also causes axial and tilting deflection of the spindle system as shown in Fig. 15.

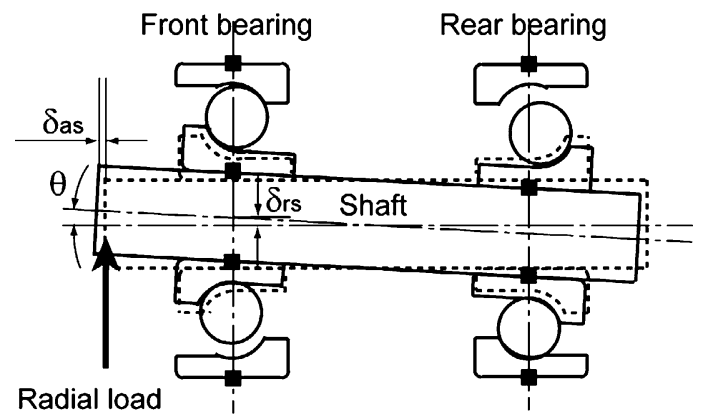

(a) Rigid preload

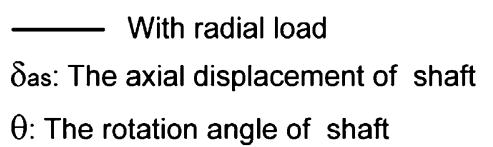

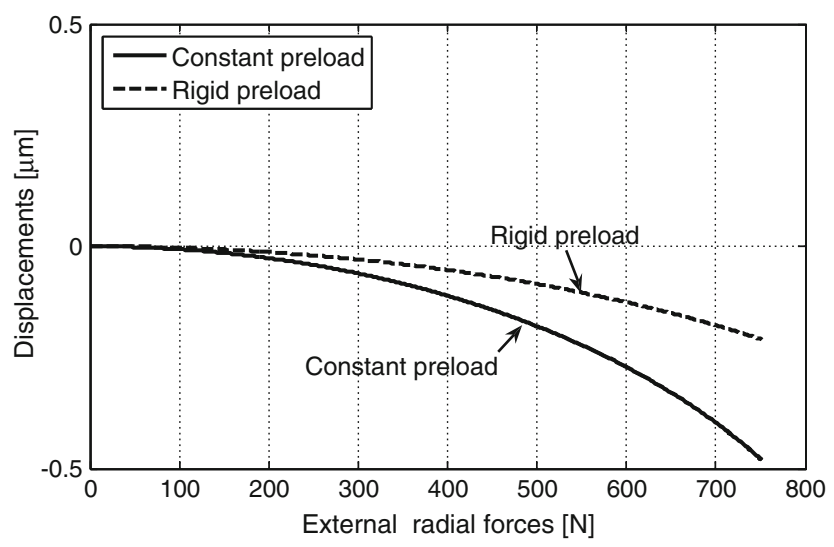

(a) Axial displacement

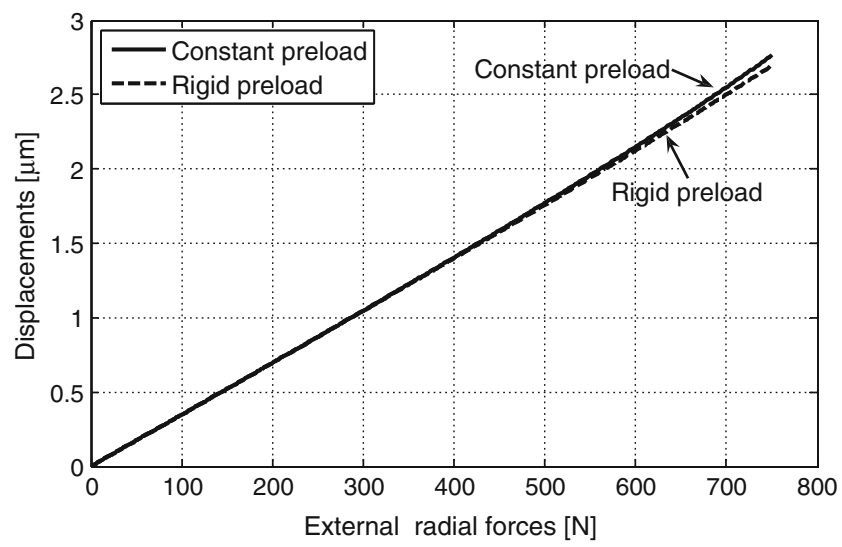

(b) Radial displacement

Fig. 16 Displacements at the spindle nose under radial loads (bearing preload: $720 \mathrm{~N}$ )

While the axial displacements change differently, the radial displacements have an identical close-to-linear relationship with radial loads, as shown in Fig. 16.

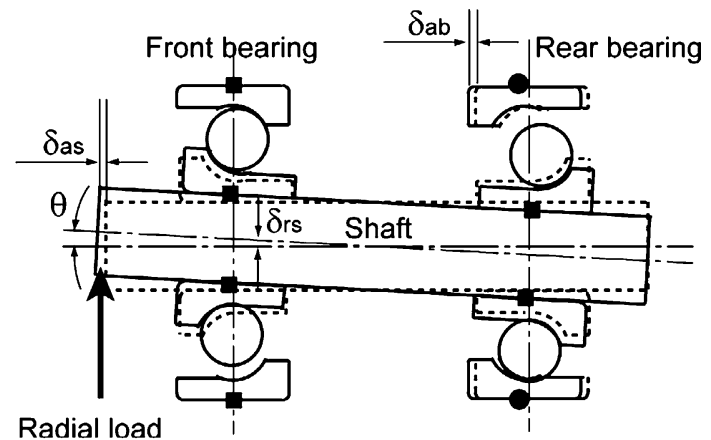

(b) Constant preload

\section{Without radial load}

$\delta a b$ : The axial displacement of rear bearing

$\delta$ rs: The radial displacement of shaft

Fig. 15 A two-bearing example - motions of shaft and bearings due to radial load 


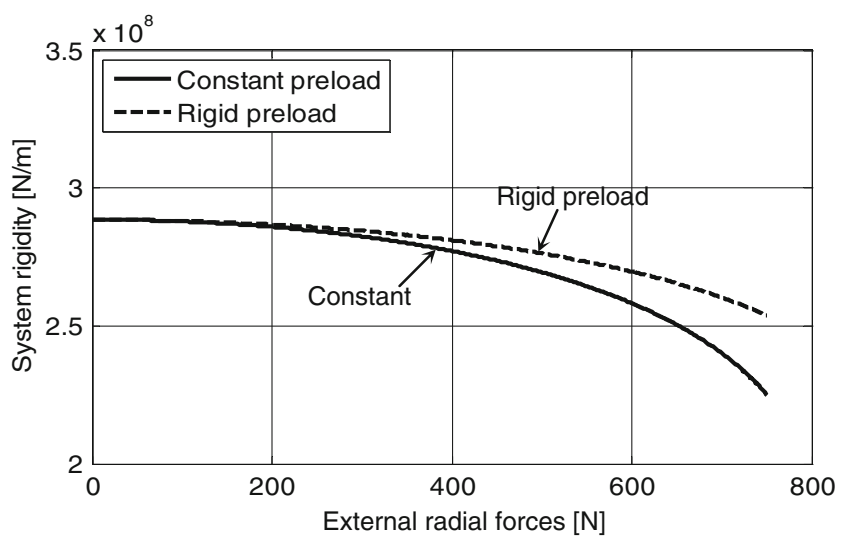

Fig. 17 Static radial stiffness at the spindle nose under radial loads (bearing preload: $720 \mathrm{~N}$ )

As the radial load is increased, the bearing stiffness is reduced, and the static radial stiffness of the spindle drops. The effect is more significant under constant preload as shown in Fig. 17.

A constant radial load of $750 \mathrm{~N}$ has been applied at the spindle nose in the radial $Y$ direction and the FRFs are predicted in the load $(Y)$ and orthogonal $(Z)$ directions in Fig. 18. The bearing stiffness changes because of variation in ball bearing contact deformations around the applied radial load; therefore, the FRFs in the two perpendicular directions vary slightly. The changes in FRFs are similar in both preload cases, while the rigid preload system loses less rigidity with radial loads. If the FRFs change significantly under large radial cutting forces, the chatter stability of the spindle system depends on the cutting conditions, which is not desirable for process-planning purposes.

\subsection{Speed effect}

During high-speed machining, the mechanical (centrifugal forces and gyroscopic moments) and thermal effects on spindle dynamics exist at the same time. It is beneficial to separate the high-speed rotation-induced changes from thermally induced preload, so that the bearing preload control strategy can be more efficient and better targeted. Thermal effects have not been considered, since they involve more complicated issues not covered in this paper. Since the speed effects from both shaft and bearing have been investigated thoroughly $[14,15]$, the main objective of this section is to compare the different preload mechanisms in terms of highspeed effects, including bearing stiffness and, the overall spindle dynamics, without considering thermal effects.

During high-speed rotation, rolling elements of bearings are subjected to extra speed-dependent loads such as centrifugal forces and gyroscopic moments, which change the bearing contact angles/loads and subsequently the bearing stiffness. The motions of the shaft and the bearing rings due to the high speed are illustrated schematically in Fig. 19.

The preload forces of both constant and rigid cases have been set to $1,900 \mathrm{~N}$. The stiffness of the bearings decreases with spindle speed rapidly, as it exceeds a certain threshold; an example for the front bearings is shown in Fig. 20. The threshold speed $(5,000 \mathrm{rev} / \mathrm{min}$ in this example) depends mainly on the value of mounting preload. The bearing stiffness in both cases decreases due to the speed effects as observed by other researchers $[15,19-21]$ while the rigid preload system maintains its stiffness better.

The axial displacements of the spindle nose are also investigated as shown in Fig. 21. In the constant preload
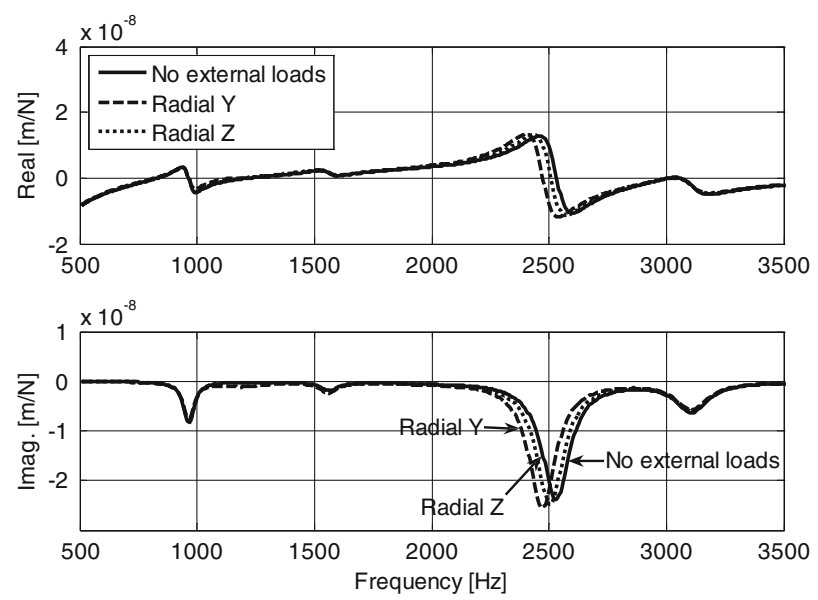

(a) Rigid preload
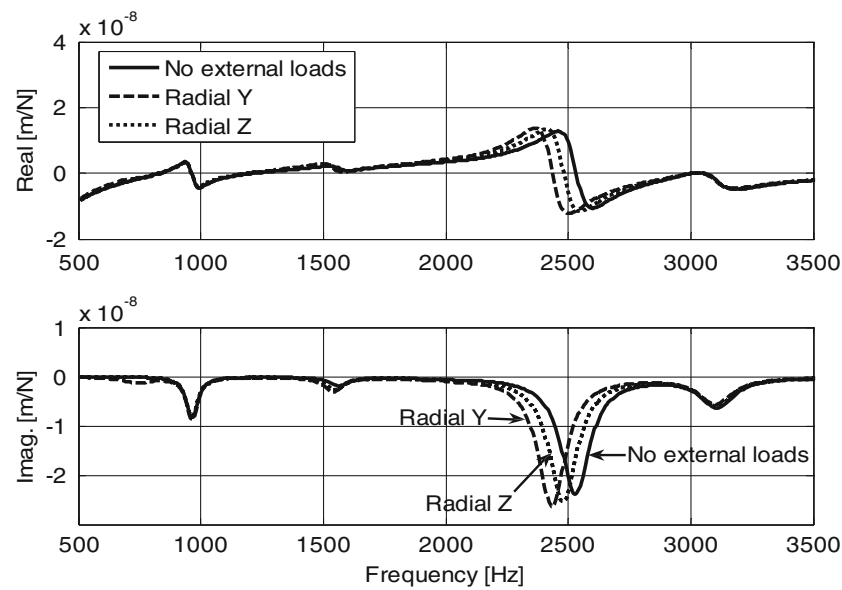

(b) Constant preload

Fig. $18 \mathrm{FRF}$ at the spindle nose in the radial direction (bearing preload: $720 \mathrm{~N}$, external radial load in the $Y$ direction: $750 \mathrm{~N}$, stationary state) 
Fig. 19 A two-bearing example - motions of shaft and bearings due to high speeds

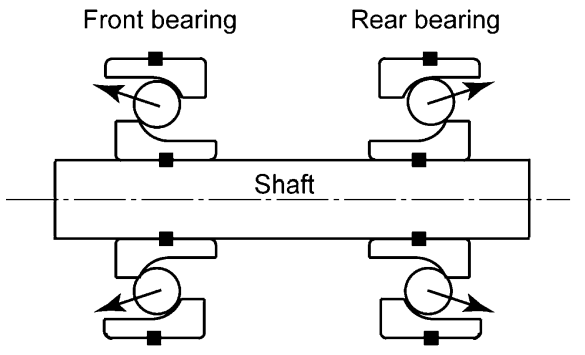

(a) Rigid preload
With speed

$\delta$ as: The axial displacement of shaft

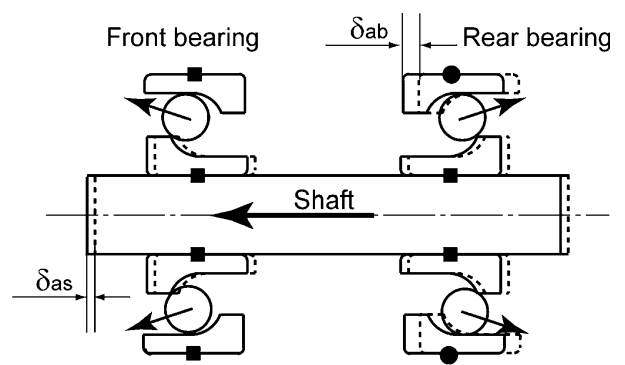

(b) Constant preload

........ Without speed

$\delta a b$ : The axial displacement of rear bearing case, the axial displacement increases significantly after a threshold speed (10,000 rev/min), which can explain the observed step changes of the axial displacement at different speeds on a high-speed machine center [22]. Bearing systems with constant preload allow rings to change their relative axial position and this is the reason why the bearing stiffness drops so dramatically. For rigid preload, the relative axial position is determined by rigid mating parts; the shaft moves slightly due to the unsymmetrical design of the spindle.

Simulated FRFs in the radial direction at the spindle nose are displayed in Fig. 22 where the rotating speed is $20,000 \mathrm{rev} / \mathrm{min}$. As the speed increases, the fourth modes are softened largely in both constant and rigid preload cases.

The chatter stability lobes are predicted using the simulated FRF at the spindle nose. The analytical chatter prediction theory of Altintas and Budak [23] is used; the results are shown in Fig. 23 using the FRF values obtained at zero spindle speed. A peak with a spindle speed of $15,320 \mathrm{rev} / \mathrm{min}$ and a depth of cut of $105.2 \mathrm{~mm}$ is chosen as a reference point to investigate the change of stability lobes

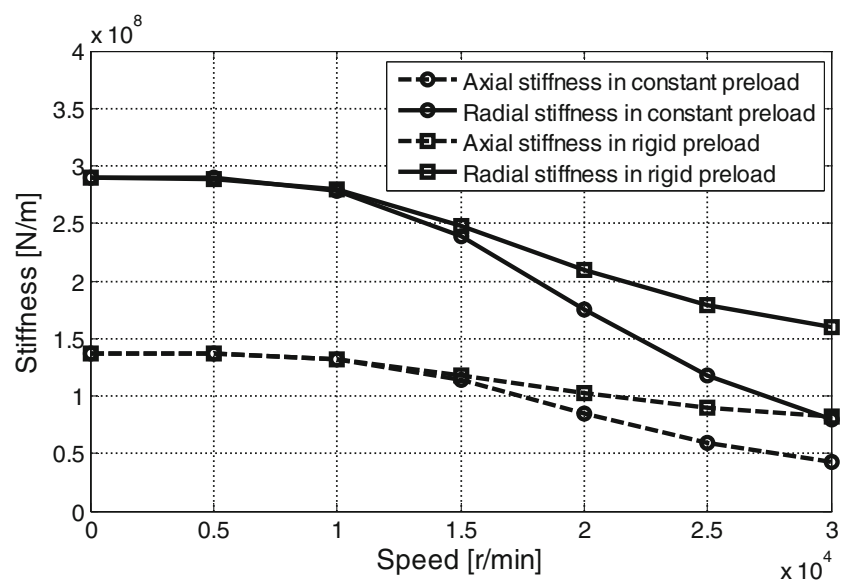

Fig. 20 Bearing stiffness changing with speeds (bearing preload: $1,900 \mathrm{~N})$ with the spindle speeds. When the spindle speed is below $10,000 \mathrm{rev} / \mathrm{min}$, the bearing stiffness in both preload cases hardly changes; the speed effects on the stability lobes can therefore be neglected. The stability lobes are calculated for each speed ranging from 10,000 to $20,000 \mathrm{rev} / \mathrm{min}$, with $1,000 \mathrm{rev} / \mathrm{min}$ increments. The changes in the stability lobes relative to the reference point are listed in Table 1 for the constant and rigid preload cases. In both preload cases, chatter-free stability pockets are shifted from high to lower spindle speeds due to the speed effect and rigid preload behaves better in maintaining the position of the reference point.

In practice, the FRFs measured in the stationary state are used to predict the stability lobes which are assumed to be speed independent. However, this assumption will cause chatter stability prediction errors in the high-speed range where the natural frequencies change. During process planning, there are two solutions to improve the prediction accuracy of the stability lobes when the speed effect cannot be neglected. The first, active, way is to increase the

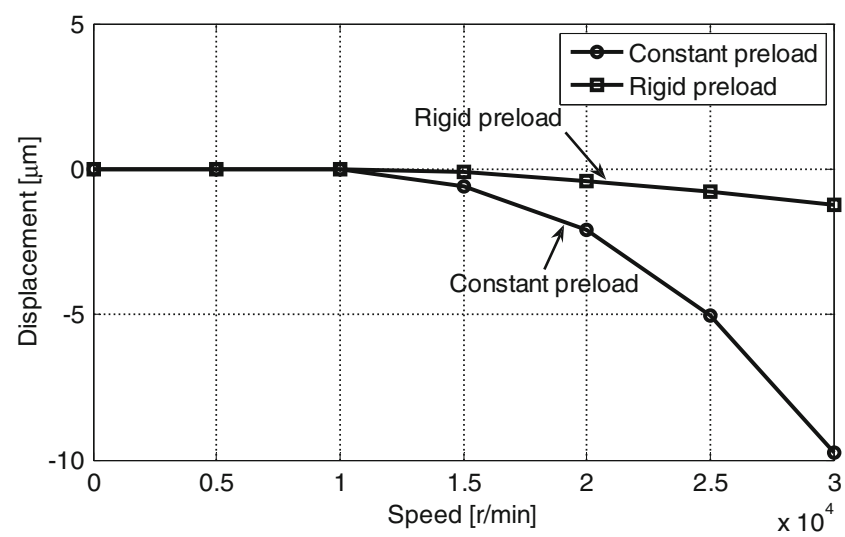

Fig. 21 Axial displacements at the spindle nose due to high speed (bearing preload: 1,900 N) 

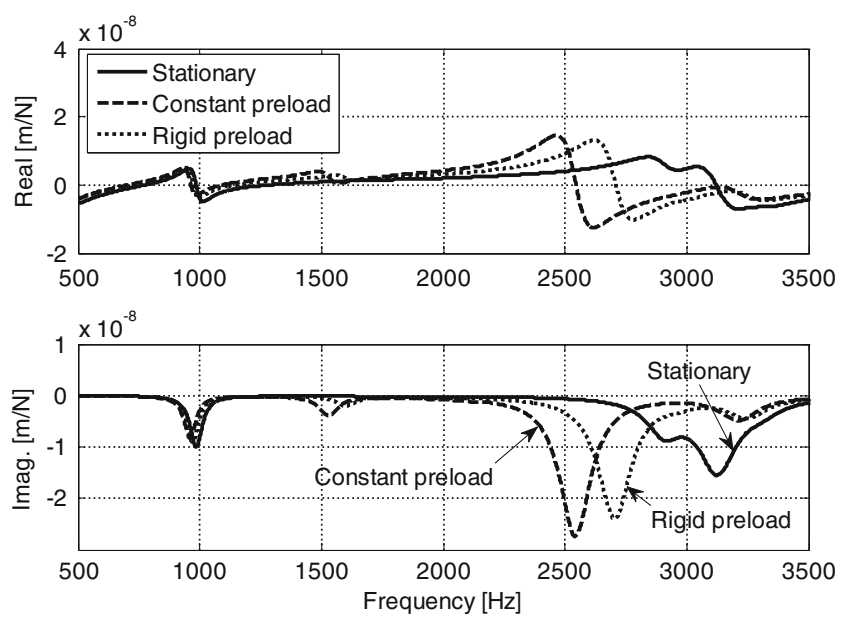

Fig. 22 FRFs at the spindle nose in the radial direction (bearing preload: $1,900 \mathrm{~N}$, spindle rotating speed: $20,000 \mathrm{rev} / \mathrm{min}$ )

preload value according to the speed and basically force the desired lobe to maintain its position with speed. However, this is done at the expenses of increased friction, decreased damping, and shorter life of bearings. Also, only specialized spindle designs with hydraulic systems allow preload modifications during rotation. The second, passive, solution is to recalculate the stability lobes and adjust process planning according to the FRFs which are identified as functions of spindle speed [24, 25].

\section{Conclusions}

Both main types of preload mechanisms for bearings - rigid and constant - have been modeled and integrated into an experimentally validated mechanical finite element model of the overall spindle system. Static and dynamic properties of spindles with both preload mechanisms have been predicted and compared analytically under various conditions. It has been shown that the overall axial rigidity of the spindle system is determined by the front bearings in the constant preload case, while the radial rigidity is similar in both preload cases.
Table 1 The change of the reference point with spindle speed in stability lobes

\begin{tabular}{lll}
\hline Spindle speed $[\mathrm{rev} / \mathrm{min}]$ & \multicolumn{2}{c}{ Speed at the reference point $[\mathrm{rev} / \mathrm{min}]$} \\
\cline { 2 - 3 } & Constant preload & Rigid preload \\
\hline 0 & 15,320 & 15,320 \\
10,000 & 15,080 & 15,090 \\
11,000 & 14,950 & 14,990 \\
12,000 & 14,790 & 14,860 \\
13,000 & 14,550 & 14,680 \\
14,000 & 14,330 & 14,470 \\
15,000 & 14,100 & 14,320 \\
16,000 & 13,840 & 14,160 \\
17,000 & 13,540 & 14,000 \\
18,000 & 13,280 & 13,820 \\
19,000 & 12,990 & 13,630 \\
20,000 & 12,680 & 13,450 \\
\hline
\end{tabular}

The external axial/radial loads have a smaller effect on systems with rigid preload than on systems with constant preload. As the spindle speed increases, lower bearing stiffness results in less stiff spindles in both preload cases and subsequently shifts chatter-free stability pockets from high to lower spindle speeds. However, the spindle system with rigid preload shows higher stiffness than the system with constant preload at high speeds; therefore, this type of preload can be preferred for high-speed spindles providing the thermally induced preload is controlled well. The speeddependent axial displacements at the spindle nose explain the step response phenomenon observed on spindles with constant preload when changing their speed.

The proposed analytical model makes it possible to predict the overall spindle dynamics and carry out detailed studies of internal displacements and bearing properties under various operating conditions. The model can be used to optimize the design of new spindles as well as to estimate the properties of already existing spindles during process planning.
Fig. 23 Predicted stability lobes without speed effects

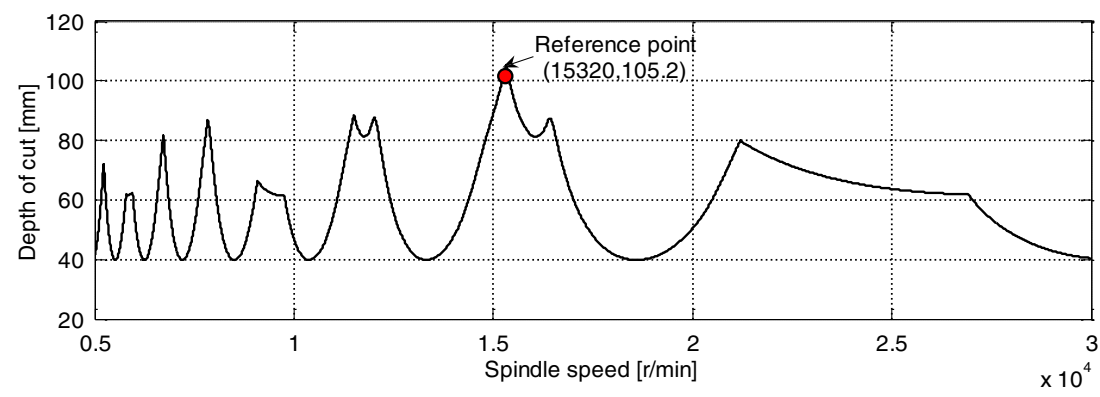


Acknowledgments This research is supported by the National Basic Research Program of China: No. 2011CB706805 for the State Key Laboratory for Manufacturing Systems Engineering, China (H. Cao), the 1M0507 grant of the Government of the Czech Republic for the Research Center of Manufacturing Technology, Prague (T. Holkup), and the Canadian NCE AUTO 21 C303 Grant (Y. Altintas).

\section{References}

1. Altintas Y (2000) Manufacturing automation-metal cutting mechanics, machine tool vibrations and CNC design. Cambridge University Press, Cambridge

2. Altintas Y, Cao Y (2005) Virtual design and optimization of machine tool spindles. Cirp Ann Manuf Techn 54:379-382

3. Li HQ, Shin YC (2004) Analysis of bearing configuration effects on high speed spindles using an integrated dynamic thermomechanical spindle model. Int J Mach Tools Manuf 44:347-364

4. Lacey SJ, Wardle FP, Poon SY (1983) High speed bearing for CNC machine tool spindles. Chartered Mech Eng 30:51-56

5. Stein JL, Tu JF (1994) A state-space model for monitoring thermally-induced preload in antifriction spindle bearings of highspeed machine-tools. J Dyn Syst T Asme 116:372-386

6. Chen JS, Chen KW (2005) Bearing load analysis and control of a motorized high speed spindle. Int J Mach Tools Manuf 45:1487-1493

7. Li HQ, Shin YC (2004) Integrated dynamic thermo-mechanical modeling of high speed spindles, part 1: model development. J Manuf Sci E T Asme 126:148-158

8. Kim SM, Lee SK (2005) Spindle housing design parameter optimization considering thermo-elastic behaviour. Int $\mathrm{J}$ Adv Manuf Tech 25:1061-1070

9. Holkup T, Cao H, Kolár P, Altintas Y, Zelený J (2010) Thermomechanical model of spindles. CIRP Annals Manufac Tech 59 (1):365-368

10. Mannan MA, Stone BJ (1998) The use of vibration measurements for quality control of machine tool spindles. Int J Adv Manuf Tech 14:889-893
11. Spiewak SA, Nickel T (2001) Vibration based preload estimation in machine tool spindles. Int J Mach Tools Manuf 41:567-588

12. Hwang YK, Lee CM (2009) Development of automatic variable preload device for spindle bearing by using centrifugal force. Int $\mathrm{J}$ Mach Tools Manuf 49:781-787

13. Jiang SY, Mao HB (2010) Investigation of variable optimum preload for a machine tool spindle. Int $\mathrm{J}$ Mach Tools Manuf 50:19-28

14. Lin CW, Tu JF, Kamman J (2003) An integrated thermomechanical-dynamic model to characterize motorized machine tool spindles during very high speed rotation. Int J Mach Tools Manuf 43:1035-1050

15. Cao YZ, Altintas Y (2004) A general method for the modeling of spindle-bearing systems. J Mech Des 126:1089-1104

16. Cao YZ, Altintas Y (2007) Modeling of spindle-bearing and machine tool systems for virtual simulation of milling operations. Int J Mach Tools Manuf 47:1342-1350

17. Jones AB (1960) A general theory for elastically constrained ball and radial roller bearings under arbitrary load and speed conditions. J Basic Eng Trans ASME 82:309-320

18. Ewins DJ (2003) Modal testing: theory, practice and application (2nd ed.). Research Studies Press, Baldock

19. Li HQ, Shin YC (2004) Integrated dynamic thermo-mechanical modeling of high speed spindles, part 2: solution procedure and validations. J Manuf Sci E T Asme 126:159-168

20. Shin YC (1992) Bearing nonlinearity and stability analysis in high-speed machining. J Eng Ind T Asme 114:23-30

21. Chen JS, Hwang YW (2006) Centrifugal force induced dynamics of a motorized high-speed spindle. Int J Adv Manuf Tech 30:10-19

22. Jedrzejewski J, Kwasny W, Modrzycki W (2008) Limiting precision distortions in spindle unit of HSC machining centre. J Mach Eng 8:44-53

23. Altintas Y, Budak E (1995) Analytical prediction of stability lobes in milling. CIRP Ann Manuf Technol 44:357-362

24. Movahhedy MR, Mosaddegh P (2006) Prediction of chatter in high speed milling including gyroscopic effects. Int J Mach Tools Manuf 46:996-1001

25. Gagnol V, Bougarrou BC, Ray P, Barra C (2007) Stability-based spindle design optimization. J Manuf Sci E T Asme 129:407-415 